\title{
Aspectos relacionados à gravidez e pós-parto de mulheres submetidas à cirurgia
}

\section{bariátrica}

\author{
Aspects related to the pregnancy and post-partition of women undergoing bariatric surgery \\ Aspectos relacionados con el embarazo y postpartición de mujeres operadas a cirugía bariátrica
}

Recebido: 02/02/2021 | Revisado: 07/02/2021 | Aceito: 08/02/2021 | Publicado: 17/02/2021

\author{
Luana Fernandes e Silva \\ ORCID: https://orcid.org/0000-0002-9174-013X \\ Universidade Federal de Minas Gerais, Brasil \\ E-mail: fernandesprofissional@ gmail.com \\ Natália Gomes Rodrigues \\ ORCID: https://orcid.org/0000-0001-7851-5643 \\ Universidade Federal de Minas Gerais, Brasil \\ E-mail: nataliagrfisica@gmail.com \\ Marina Dayrell de Oliveira Lima \\ ORCID: https://orcid.org/0000-0002-8880-1659 \\ Universidade Federal de Minas Gerais, Brasil \\ E-mail: marina_dayrell@hotmail.com \\ Thales Philipe Rodrigues da Silva \\ ORCID: https://orcid.org/0000-0002-7115-0925 \\ Universidade Federal de Minas Gerais, Brasil \\ E-mail: thalesphilipe27@ hotmail.com \\ Lauro Pinheiro Ferreira de Araújo \\ ORCID: https://orcid.org/0000-0002-8074-7547 \\ Hospital Santa Rita, Brasil \\ E-mail: araujolauro@hotmail.com \\ Anna Luísa Marques Mariano Roque \\ ORCID: https://orcid.org/0000-0003-4075-5742 \\ Universidade Federal de Minas Gerais, Brasil \\ E-mail: anna.mmroque@gmail.com \\ Fernanda Penido Matozinhos \\ ORCID: https://orcid.org/0000-0003-1368-4248 \\ Universidade Federal de Minas Gerais, Brasil \\ E-mail: nandapenido@hotmail.com
}

\begin{abstract}
Resumo
Objetivo: Analisar os aspectos sociodemográficos, gineco-obstétricos, clínicos e comportamentais relacionados à gravidez e pós-parto de mulheres submetidas à cirurgia bariátrica. Método: Trata-se de uma coorte prospectiva, com 33 mulheres submetidas à cirurgia bariátrica, em um hospital privado de Minas Gerais. A coleta de dados ocorreu por meio de um questionário semiestruturado, aplicado por meio de ligações telefônicas. As análises foram realizadas usando-se o software Stata, versão 14.0. Resultados: O perfil gineco-obstétrico demonstrou que 78,79\% das mulheres apresentaram melhora no desempenho sexual após a cirurgia bariátrica. Destas, $51,52 \%$ tiveram como via de nascimento a cesariana. 96,97\% das mulheres não relataram complicações após a gestação, 76\% conseguiram amamentar e 69,57\% não tiveram intercorrências na amamentação. $81,25 \%$ não informaram nenhuma complicação de saúde do bebê. O perfil clínico evidenciou que 63,64\% não foram acompanhadas por nutricionista após a cirurgia e 78,79\% não tiveram acompanhamento por psicólogo ou psiquiatra. 96,97\% não tiveram diagnóstico de Hipertensão Arterial Sistêmica após a cirurgia bariátrica e $100 \%$ não foram diagnosticadas de Diabetes Mellitus após o procedimento cirúrgico. Por fim, o perfil comportamental demonstrou que 78,79\% das mulheres não praticavam atividade física nos últimos 3 meses, 66,67\% não consumiam bebida alcoólica e $87,88 \%$ não eram tabagistas. Conclusão: O procedimento de cirurgia bariátrica está relacionado a desfechos positivos, portanto, a manutenção destes fatores está relacionada à forma de encarar os novos hábitos de vida e o acompanhamento multiprofissional. Apesar dos benefícios comprovados do procedimento cirúrgico, o mesmo pode levar a complicações clínicas e nutricionais maternas e fetais.
\end{abstract}

Palavras-chave: Gravidez; Período pós-parto; Cirurgia bariátrica; Obesidade.

\section{Abstract}

Objective: To analyze the sociodemographic, gynecological-obstetric, clinical and behavioral aspects related to pregnancy and postpartum women submitted to bariatric surgery. Method: This is a prospective cohort, with 33 women undergoing bariatric surgery, in a private hospital in Minas Gerais. Data collection took place through a semi- 
structured questionnaire, applied through telephone calls. The analyzes were performed using the Stata software, version 14.0. Results: The gynecological-obstetric profile showed that $78.79 \%$ of women showed improvement in sexual performance after bariatric surgery. Of these, 51.52\% had a cesarean section as their route of birth. $96.97 \%$ of women did not report complications after pregnancy, $76 \%$ were able to breastfeed and $69.57 \%$ had no complications during breastfeeding. $81.25 \%$ did not report any health complications of the baby. The clinical profile showed that $63.64 \%$ were not followed up by a nutritionist after the surgery and $78.79 \%$ were not followed up by a psychologist or psychiatrist. $96.97 \%$ were not diagnosed with Systemic Arterial Hypertension after bariatric surgery and $100 \%$ were not diagnosed with Diabetes Mellitus after the surgical procedure. Finally, the behavioral profile showed that $78.79 \%$ of women did not practice physical activity in the last 3 months, $66.67 \%$ did not consume alcohol and $87.88 \%$ were not smokers. Conclusion: The bariatric surgery procedure is related to positive outcomes, therefore, the maintenance of these factors is related to the way of facing new life habits and multiprofessional monitoring. Despite the proven benefits of the surgical procedure, it can lead to maternal and fetal clinical and nutritional complications.

Keywords: Pregnancy; Postpartum period; Bariatric surgery; Obesity.

\section{Resumen}

Objetivo: Analizar los aspectos sociodemográficos, ginecológico-obstétricos, clínicos y conductuales relacionados con el embarazo y el posparto de mujeres sometidas a cirugía bariátrica. Método: Se trata de una cohorte prospectiva, con 33 mujeres sometidas a cirugía bariátrica, en un hospital privado de Minas Gerais. La recolección de datos se realizó mediante un cuestionario semiestructurado, aplicado a través de llamadas telefónicas. Los análisis se realizaron utilizando el software Stata, versión 14.0. Resultados: El perfil ginecológico-obstétrico mostró que el 78,79\% de las mujeres mostró mejoría en el desempeño sexual después de la cirugía bariátrica. De estos, el 51,52\% tuvo una cesárea como vía de nacimiento. El 96,97\% de las mujeres no reportó complicaciones después del embarazo, el 76\% pudo amamantar y el 69,57\% no tuvo complicaciones durante la lactancia. El 81,25\% no informó ninguna complicación de salud del bebé. El perfil clínico mostró que el 63,64\% no fue seguido por un nutricionista después de la cirugía y el $78,79 \%$ no fue seguido por un psicólogo o psiquiatra. El 96,97\% no fue diagnosticado de Hipertensión Arterial Sistémica después de la cirugía bariátrica y el $100 \%$ no fue diagnosticado con Diabetes Mellitus después del procedimiento quirúrgico. Finalmente, el perfil conductual mostró que el 78,79\% de las mujeres no practicó actividad física en los últimos 3 meses, el 66,67\% no consumía alcohol y el 87,88\% no era fumador. Conclusión: El procedimiento de cirugía bariátrica se relaciona con resultados positivos, por tanto, el mantenimiento de estos factores está relacionado con la forma de afrontar los nuevos hábitos de vida y el seguimiento multiprofesional. A pesar de los beneficios probados del procedimiento quirúrgico, puede conducir a complicaciones clínicas y nutricionales maternas y fetales.

Palabras clave: Embarazo; Período pós-parto; Cirugía bariátrica; Obesidad.

\section{Introdução}

A obesidade é uma condição crônica multifatorial, com origens genéticas, comportamentais e ambientais (Hruby \& Hu, 2015).

A obesidade possui alta prevalência mundial e constitui como fator de risco para diversas doenças (Amaral, 2016). Resultados de duas importantes pesquisas do Instituto Brasileiro de Geografia e Estatística (IBGE), realizadas entre os anos de 2003 a 2019, a Pesquisa de Orçamentos Familiares (POF) e a Pesquisa Nacional de Saúde (PNS), mostraram que a proporção de obesos na população com 20 anos ou mais de idade no Brasil passou de 12,2\% para 26,8\%. Nesse período, a prevalência da obesidade entre mulheres aumentou de $14,5 \%$ para 30,2\% e, entre os homens, de 9,6\% para 22,8\% (Brasil, 2020).

O manejo da obesidade é bastante complexo e de caráter individualizado e leva em consideração as condições físicas, clínicas e psicológicas do paciente, envolvendo abordagens de controle variadas, tais como processos nutricionais, físicos e aspectos cirúrgicos, levando em consideração o padrão de consumo alimentar e o gasto energético (Rosales, 2020; Malta, Silva, Tonaco, Freitas, \& Velasquez-Melendez, 2019).

Um dos métodos para tratar indivíduos com obesidade é a cirurgia bariátrica, a qual resulta em perda de peso significativa, melhoria das comorbidades decorrentes do excesso de peso e otimização da qualidade de vida (Raoof et al., 2015; Major et al., 2015). Ela auxilia na recuperação de funções rotineiras da vida e da autoestima, por meio da mudança de estilo de vida promovida pelos novos hábitos pós-cirúrgicos, já que muitos indivíduos com obesidade grave não conseguem nem mesmo 
fazer algumas necessidades fisiológicas sozinhos. Assim, oferece uma melhor perspectiva de vida, melhorando a saúde física e mental (Sousa \& Johann, 2014; Oliveira, Filho, Venturini, Castro, \& Ferreira, 2018).

A intervenção cirúrgica representa uma das possibilidades eficazes para o tratamento de pessoas com obesidade, respeitando o cumprimento dos seguintes critérios: indivíduos que não responderam ao tratamento clínico longitudinal, que inclui orientação e apoio para mudança de hábitos, reeducação alimentar, atenção psicológica, prescrição de Atividade Física (AF) e, se necessário, farmacoterapia (Brasil, 2013). Refere-se a um conjunto de técnicas cirúrgicas utilizadas para diminuir o peso corporal e melhorar o estado metabólico do indivíduo (Ferrer, Mundaca, Ledezma, \& Carvajal, 2016). É o método de referência para o tratamento da obesidade, devido à durabilidade e ritmo da perda de peso, redução dos sintomas e remissão de doenças concomitantes (Różańska-walędziak, Walędziak, Bartniki, \& Kacperczyk-Bartnik, 2020), além de melhorar a expectativa e a qualidade de vida dos pacientes (Petrucciani, 2020).

O Brasil é considerado o segundo país do mundo em número de cirurgias bariátricas realizadas e as mulheres representam 76\% dos pacientes (Sociedade Brasileira de Cirurgia Bariátrica e Metabólica [SBCBM], 2018). A maior parte das mulheres que realizam Cirurgia Bariátrica (CB) estão em idade reprodutiva, já que a obesidade está relacionada a vários riscos à saúde das mulheres, como diminuição da fertilidade e complicações na gestação e pós-parto (Eppel \& Christian, 2020).

O procedimento cirúrgico pode ser um método para alcançar a perda de peso, que pode otimizar a vida sexual, a regularização da menstruação e da ovulação, a fertilidade e a redução das complicações associadas à obesidade na gestação e no pós-parto (Ferrer et al., 2016). Promove aumento da satisfação com a imagem corporal, contribuindo para melhora da autoestima e do convívio social, refletindo positivamente na vivência da sexualidade (Mariano, 2014).

Embora a perda de peso mediada pela cirurgia tenha um efeito positivo no resultado da gravidez, o procedimento pode estar associado a complicações, como deficiência de micronutrientes, anemia por deficiência de ferro ou vitamina B12, síndrome de dumping, complicações cirúrgicas, como hérnias internas e filhos pequenos para a idade gestacional (IG), possivelmente devido à desnutrição materna (Falcone, 2018).

A CB parece ser uma intervenção promissora na redução das complicações perinatais relacionadas à obesidade. No entanto, apesar dos benefícios comprovados do procedimento cirúrgico, sabe-se que o mesmo pode levar ao risco de subnutrição da mãe ou feto durante a gravidez, principalmente pelo risco de desenvolver deficiências nutricionais de ferro, ácido fólico, vitamina B12, cálcio e vitamina D, que podem ocasionar complicações fetais como nascimento prematuro, baixo peso ao nascer, hipocalcemia neonatal ou raquitismo, retardo mental fetal e defeitos do tubo neural (Poitou, 2007).

A produção científica brasileira sobre os fatores referentes à gravidez e pós-parto de mulheres submetidas à CB é relativamente recente. Compreender as mudanças que o procedimento cirúrgico e a gravidez promovem no organismo da mulher é, portanto, fundamental para desenvolver estratégias efetivas para reduzir os impactos negativos na gestação e no período pósparto.

Soma-se, a este fato, a escassez de estudos em locais específicos, como em instituições de saúde privadas e com amostras específicas, como a de mulheres que engravidaram após o procedimento de cirurgia bariátrica.

O presente estudo objetivou analisar aspectos sociodemográficos, gineco-obstétricos, clínicos e comportamentais relacionados à gravidez e pós-parto de mulheres submetidas à CB.

A hipótese deste estudo é que embora o procedimento cirúrgico leve a melhorias clínicas, na ovulação e na taxa de gestação, deve-se levar em consideração os riscos nutricionais e possíveis complicações da cirurgia, como obstrução do intestino delgado, hérnias internas e colelitíase, que podem resultar na evolução desfavorável da gestação (Coelho et al., 2020; Melo \& Melo, 2017). 
No entanto, os estudos destacam a relevância do acompanhamento multiprofissional nos períodos pré, trans e pós cirúrgico, e que ainda não está claramente definido qual o intervalo temporal que deve decorrer entre a realização da cirurgia bariátrica e uma gravidez. É consensual que a gravidez não deve ocorrer no período de maior perda de peso, ou seja, até 12 a 18 meses após a cirurgia (Costa, Bombas, Souto, \& Freitas, 2020).

\section{Métodos}

\section{Desenho e local do estudo}

Trata-se de um estudo de natureza quantitativa. Foi realizada uma coorte prospectiva, com mulheres submetidas à CB no período de 2012 a 2014, em um hospital privado de Minas Gerais, Brasil.

A manipulação estatística permite comprovar as relações dos fenômenos entre si, e obter generalizações sobre sua natureza, ocorrência ou significado (Pereira, Shitsuka, Parreira, \& Shitsuka, 2018). Estudos de coorte fazem parte do grupo de estudos observacionais de cunho epidemiológico que se propõem a observar, em uma população previamente definida, qual será a incidência de determinada doença ou fenômeno relacionado à saúde. Podem ser usados para avaliação de desfechos múltiplos, são menos sujeitos a viéses de seleção do que os estudos de caso-controle e permitem ainda que várias exposições possam ser avaliadas (Oliveira \& Parente, 2010).

\section{População e amostra do estudo}

A amostra foi constituída por 33 mulheres que engravidaram no período de seguimento de 2016 a 2019.

Em relação aos critérios de inclusão, participaram desta pesquisa as mulheres com 18 ou mais anos de idade que conseguiram engravidar após a CB.

Foram excluídas as mulheres que se recusaram a participar do estudo ou que não atenderam a ligação telefônica.

\section{Coleta de dados}

A coleta de dados foi realizada no período de 2016 a 2019, por meio de um questionário semiestruturado elaborado pelos pesquisadores, com variáveis socioeconômicas, gineco-obstétricas, clínicas e comportamentais, aplicado por meio de ligações telefônicas aos pacientes.

As ligações foram realizadas por profissionais treinados e ocorreu no período de três meses no início de cada ano da coorte, em horários diferentes nos turnos da manhã, tarde e noite, pelo menos três vezes em cada turno, para cada participante.

\section{Variáveis e critérios adotados}

As variáveis foram apresentadas em unidades/categorias, incluindo as variáveis socioeconômicas, gineco-obstétricas, clínicas e comportamentais (Quadro 1). 
Quadro 1 - Descrição das variáveis individuais - Belo Horizonte e Contagem - Minas Gerais - 2016 a 2019.

\begin{tabular}{|c|c|c|}
\hline Variável & Unidade/categoria & Classificação \\
\hline \multicolumn{3}{|l|}{ Socioeconômica } \\
\hline Idade & Anos & Discreta \\
\hline Escolaridade & $\begin{array}{l}\text { Ensino superior; ensino médio; ensino fundamental; } \\
\text { educação primária }\end{array}$ & Ordinal \\
\hline Renda média domiciliar & $\begin{array}{c}\text { Até } 1 \text { salário mínimo; de } 1 \text { a } 3 \text { salários mínimos; de } 3 \text { a } 5 \\
\text { salários mínimos; mais que } 5 \text { salários mínimos }\end{array}$ & Ordinal \\
\hline Situação conjugal & Vive sem companheiro(a); vive com companheiro(a) & Nominal \\
\hline \multicolumn{3}{|l|}{ Gineco-obstétrica } \\
\hline $\begin{array}{l}\text { Melhora no desempenho sexual após a } \\
\text { cirurgia bariátrica }\end{array}$ & Sim; não & Nominal \\
\hline $\begin{array}{c}\text { Tempo que conseguiu engravidar após a } \\
\text { cirurgia bariátrica }\end{array}$ & Meses & Discreta \\
\hline Realização de pré-natal & Sim; não & Nominal \\
\hline $\begin{array}{l}\text { Quantidade de consultas de pré-natal } \\
\text { realizadas }\end{array}$ & Número de consultas & Contínua \\
\hline Via de nascimento & Vaginal; cesárea & Nominal \\
\hline Complicação durante e após a gestação & Sim; não & Nominal \\
\hline $\begin{array}{l}\text { Tipo de complicação durante e após a } \\
\text { gestação }\end{array}$ & $\begin{array}{l}\text { Anemia; deficiência de vitaminas; diabetes gestacional; } \\
\text { hipertensão; hipoglicemia; sangramento; toxoplasmose; } \\
\text { sopro no coração; prolapso de cordão. }\end{array}$ & Nominal \\
\hline Amamentação & Sim; não & Nominal \\
\hline $\begin{array}{l}\text { Problema durante o processo de } \\
\text { amamentação }\end{array}$ & Sim; não & Nominal \\
\hline
\end{tabular}




\begin{tabular}{|c|c|c|}
\hline $\begin{array}{l}\text { Tipo de problema durante o processo de } \\
\text { amamentação }\end{array}$ & $\begin{array}{l}\text { Fissura; pouco leite; ingurgitamento; não teve leite; o } \\
\text { leite secou. }\end{array}$ & Nominal \\
\hline Complicação com o bebê & Sim; não & Nominal \\
\hline Tipo de complicação com o bebê & $\begin{array}{l}\text { Bradicardia; dispnéia; espinha bífida; síndrome do } \\
\text { intestino curto; baixo peso. }\end{array}$ & Nominal \\
\hline \multicolumn{3}{|l|}{ Clínica } \\
\hline Índice de Massa Corporal Atual & $\mathrm{kg} / \mathrm{m}^{2}$ & Contínua \\
\hline Ganho de peso após ter emagrecido & Sim; não & Nominal \\
\hline Acompanhamento por nutricionista & Sim; não & Nominal \\
\hline $\begin{array}{c}\text { Acompanhamento por psicólogo e/ou } \\
\text { psiquiatra }\end{array}$ & Sim; não & Nominal \\
\hline $\begin{array}{l}\text { Diagnóstico de Hipertensão Arterial } \\
\text { Sistêmica após a cirurgia bariátrica }\end{array}$ & Sim; não & Nominal \\
\hline $\begin{array}{c}\text { Diagnóstico de Diabetes Mellitus após a } \\
\text { cirurgia bariátrica }\end{array}$ & Sim; não & Nominal \\
\hline \multicolumn{3}{|l|}{ Comportamental } \\
\hline $\begin{array}{c}\text { Prática de atividade física nos últimos } \\
\text { três meses }\end{array}$ & Sim; não & Nominal \\
\hline Consumo de álcool & Sim; não & Nominal \\
\hline Tabagismo & Sim; não; ex-fumante & Nominal \\
\hline
\end{tabular}

Fonte: Autores, para fins de estudo.

\section{Análise dos dados}

A análise dos dados foi realizada por meio da distribuição de frequências absolutas e relativas. Para as variáveis contínuas, foi verificada a simetria de distribuição pelo teste de Shapiro-Wilk. Os resultados foram apresentados por meio de média e desvio padrão (DP).

Os resultados foram apresentados no formato de tabelas. Ressalta-se que os totais dos números das variáveis podem variar, devido à ausência de alguns dados para as variáveis estudadas. 
As análises foram realizadas usando-se o software Stata, versão 14.0.

O Índice de Massa Corporal (IMC), indicador amplamente usado em análises de situações relacionadas ao peso, foi calculado pela fórmula (peso [kg]/altura $\left[\mathrm{m}^{2}\right]$ ), (lopes et al., 2019). O excesso de peso foi diagnosticado quando o IMC alcançou um valor igual ou superior a $25 \mathrm{~kg} / \mathrm{m}^{2}$, enquanto a obesidade foi determinada com valor de IMC igual ou superior a $30 \mathrm{~kg} / \mathrm{m}^{2}$ (Brasil, 2020).

\section{Considerações Éticas}

Por se tratar de entrevistas via telefone, o consentimento livre e esclarecido foi substituído pelo consentimento verbal, obtido por ocasião dos contatos telefônicos com os entrevistados, os quais foram esclarecidos quanto à confidencialidade e ao anonimato dos dados e que eles seriam utilizados apenas para fins de pesquisa. A todos os entrevistados foi fornecido um número de telefone para esclarecimento de dúvidas.

O estudo foi aprovado pelo Comitê de Ética em Pesquisa da Universidade Federal de Minas Gerais: CAAE52657115.2 .0000 .5149 .

\section{Resultados}

A amostra deste estudo foi constituída por 33 mulheres, com idade média de 33 anos (DP = 4,63). Das 33 gestantes, $66,67 \%$ possuíam ensino médio completo, $87,88 \%$ viviam com companheiro e $59,38 \%$ possuíam renda média domiciliar de 1 a 3 salários mínimos (Tabela 1).

Tabela 1 - Perfil sociodemográfico das gestantes submetidas à cirurgia bariátrica, Contagem, Minas Gerais, Brasil, $2016-2019$.

\begin{tabular}{|lc|}
\hline PERFIL SOCIODEMOGRÁFICO & n $(\%)$ \\
\hline Idade* & $33(4,63)$
\end{tabular}

\section{Escolaridade}

Ensino superior

Ensino médio

Ensino fundamental

\section{Estado conjugal}

Vive sem companheiro

Vive com companheiro

\section{Renda média domiciliar}

Até 1 salário mínimo

1-| 3 salários mínimos

3-| 5 salários mínimos

Mais de 5 salários mínimos

$1(3,13)$

Nota: *Média $( \pm \mathrm{DP})$. Fonte: Autores, para fins de estudo. 
A idade média demonstra que a amostra é composta por mulheres em idade fértil, sendo que a maioria delas vivem com companheiro.

A Tabela 2 apresenta os dados referentes ao perfil gineco-obstétrico das mulheres da amostra.

Destaca-se que 78,79\% das mulheres apresentaram melhora no desempenho sexual após a CB e engravidaram com tempo médio de 31 meses ( $\mathrm{DP}=18,12$ ). Ademais, a maioria dessas mulheres relataram ter realizado consultas de pré-natal, com uma maior prevalência pela via de nascimento cesariana.

No que tange às complicações durante a gestação, houveram relatos de $21,21 \%$ das mulheres com algum tipo de complicação, sendo a anemia a mais prevalente $(27,27 \%)$, conforme detalhado na Tabela 2.

Verificou-se que 96,97\% das mulheres submetidas à CB não relataram complicações após a gestação.

A maioria das mulheres conseguiu amamentar e não relataram intercorrências durante o processo de amamentação. A fissura no bico $(28,57 \%)$ e o fato de não ter tido leite $(28,57 \%)$ foram as complicações mais prevalentes relatadas.

Em relação às complicações com o bebê, observou-se que a maioria não informou nenhuma complicação de saúde. A dificuldade respiratória $(33,33 \%)$ foi a complicação mais relatada.

Tabela 2 - Perfil gineco-obstétrico das gestantes submetidas à cirurgia bariátrica, Contagem, Minas Gerais, Brasil, $2016-2019$.

\begin{tabular}{lr}
\hline PERFIL GINECO-OBSTÉTRICO & $\mathbf{n}(\%)$ \\
\hline Melhora no desempenho sexual após a cirurgia bariátrica & $26(78,79)$ \\
Sim & $7(21,21)$ \\
Não & $31(18,12)$ \\
Tempo (em meses) que conseguiu engravidar após a cirurgia bariátrica** & $7(21,21)$ \\
Realização de pré-natal & $26(78,79)$ \\
Não responderam & $10,09(3,65)$ \\
Sim &
\end{tabular}

Via de nascimento

Não responderam

Vaginal

Cesária

Complicação durante a gestação

Não

Sim

Tipo de complicação durante a gestação

Anemia

Deficiência de vitaminas 
Hipoglicemia

Toxoplasmose

Sopro no coração

\section{Complicação após a gestação}

Sim

Não

Tipo de complicação após a gestação

Não responderam

Hipertensão Arterial Sistêmica

\section{Amamentação}

$\operatorname{Sim}$

Não

\section{Problema durante o processo de amamentação}

Sim

Não

Tipo de problema durante o processo de amamentação

Fissura e pouco leite

Fissura no bico

Ingurgitamento e fissura

$1(14,29)$

Leite secou

Não teve leite

\section{Complicação com o bebê}

$\operatorname{Sim}$

Não

Tipo de complicação com o bebê

Bradicardia

Dificuldade respiratória

Espinha bífida

Síndrome do intestino curto

$1(16,16)$ 
Destaca-se a melhora no desempenho sexual após o procedimento cirúrgico, fato este que impacta nas chances de engravidar após a cirurgia. É importante enfatizar ainda a prevalência da via de nascimento cesária, condição que não tem relação com o procedimento de cirurgia bariátrica. Foi demonstrado que a maioria dessas mulheres não tiveram complicações antes e após a gestação, não relataram complicações com o bebê e não apresentaram problemas durante o processo de amamentação.

Em relação ao perfil clínico, a Tabela 3 demonstra a média do IMC pós-cirúrgico das mulheres e o acompanhamento por profissional psicólogo ou psiquiatra e por um profissional nutricionista no pós-operatório.

Tabela 3 - Perfil clínico das gestantes submetidas à cirurgia bariátrica, Contagem, Minas Gerais, Brasil, 2016 -2019.

\begin{tabular}{lc}
\hline PERFIL CLÍNICO & n (\%) \\
\hline IMC* & $29,21(5,74)$
\end{tabular}

Ganho de peso após ter emagrecido

$\operatorname{Sim}$

Não

Acompanhamento com nutricionista

Sim

Não

Acompanhamento por profissional psicólogo e/ou psiquiatra

Sim

Não

Diagnóstico de Hipertensão Arterial Sistêmica após a cirurgia bariátrica

Sim

Não

$32(96,97)$

Diagnóstico de Diabetes Mellitus após a cirurgia bariátrica

Sim

Não

$33(100)$

A classificação do IMC demonstra uma média de mulheres com sobrepeso após o procedimento cirúrgico e que a maioria dessas mulheres não tiveram acompanhamento por profissional psicólogo ou psiquiatra e por um profissional nutricionista no pós-operatório.

Quanto ao perfil comportamental, observa-se na Tabela 4 a prática de atividade física nos últimos três meses, o consumo de bebida alcoólica e tabagismo. 
Tabela 4 - Perfil comportamental das gestantes submetidas à cirurgia bariátrica, Contagem, Minas Gerais, Brasil, $2016-2019$.

\begin{tabular}{ll}
\hline PERFIL COMPORTAMENTAL & n $(\%)$
\end{tabular}

\section{Prática de atividade física nos últimos três meses}

$\operatorname{Sim}$

Não

\section{Consumo de bebida alcoólica}

Sim

Não

Tabagismo

Não

Ex-fumante

Fonte: Autores, para fins de estudo.

A maioria da amostra não praticava atividade física ou esporte nos últimos três meses, não consumia bebidas alcoólicas e não era tabagista.

\section{Discussão}

Este estudo revelou perfis sociodemográfico, gineco-obstétrico, clínico e comportamental associados à gravidez e ao pós-parto de mulheres submetidas à CB.

A idade média das mulheres que realizaram o procedimento cirúrgico, no presente estudo, foi de 33 anos. Pesquisas revelam que a busca pela $\mathrm{CB}$, na maioria dos casos, parte das mulheres com obesidade em idade fértil, que procuram uma melhora na fertilidade e, consequentemente, maior facilidade para engravidar, após a cirurgia (Johansson et al., 2015; Lesko \& Peaceman, 2012; Roos et al., 2013; Santo, Riccioppo \& Cecconello, 2010).

Em relação ao perfil gineco-obstétrico, esta pesquisa demonstrou que a maioria das mulheres apresentaram melhora no desempenho sexual após o procedimento cirúrgico. As alterações causadas no corpo, devido à obesidade, e o fato de se sentirem fora do padrão estético social podem gerar, nas mulheres, sentimentos de vergonha do corpo e, devido a isso, preferem isolar-se e não encarar o parceiro, seja por medo ou insegurança, ficando mais difícil finalizar o ato sexual, uma vez que o cansaço físico e a dificuldade de mobilidade se fazem presentes na maioria desses casos (Mariano, 2014).

No que diz respeito à via de nascimento, os resultados deste estudo destacaram que mais da metade das mulheres foram submetidas à cesariana. Pesquisa conduzida por Sheiner et al. (2004) evidenciou um aumento do número de cesarianas em mulheres submetidas à $\mathrm{CB}$, fato este que os autores atribuíram à excessiva preocupação da equipe médica em relação aos desfechos do parto via vaginal. No entanto, não foram evidenciadas complicações em mulheres que engravidaram após a CB, independentemente da via de nascimento. Alerta-se que a CB não deve ser indicação absoluta de cesariana (Febrasgo, 2014).

No que tange às complicações durante a gestação, os dados deste trabalho evidenciaram a anemia como o principal problema apresentado pelas mulheres. As mulheres que engravidam após a bariátrica podem ter deficiências nutricionais de ferro, ácido fólico, vitamina B12, cálcio e vitamina D, as quais podem ocasionar complicações fetais, como nascimento prematuro, baixo peso ao nascer, hipocalcemia neonatal ou raquitismo, retardo mental fetal e defeitos do tubo neural (Pinheiro, 2014). 
Ainda de acordo com este estudo, a maioria das gestantes submetidas à CB amamentaram, sendo que a minoria destas apresentaram problemas durante a amamentação. Em pesquisa desenvolvida por Wojcicki (2011), a obesidade se associou negativamente às práticas de amamentação. O autor enfatizou a associação entre sobrepeso e obesidade com a diminuição da lactogênese ou falha do processo da amamentação.

Ainda como resultados deste trabalho, evidenciou-se que a média do IMC das mulheres após a CB apresenta sobrepeso e que mais da metade das mulheres ganharam peso após terem emagrecido. Para Beleli et al. (2011), é evidente a dificuldade encontrada pelas mulheres para manter o peso após a CB. O procedimento cirúrgico é considerado eficaz para a perda de peso, porém, o reganho de peso após dois anos tem sido muito observado. Há pacientes que tendem a recuperar peso a partir do segundo ano de cirurgia devido à dificuldade em manter as mudanças estabelecidas em seus estilos de vida (Franques, Pacheco, Belfort, \& Gomes, 2011, p. 264).

Revelou-se, ainda, nesta pesquisa, inexpressivo acompanhamento por profissionais psiquiatra, psicólogo e nutricionista após a CB. Estudos destacam a importância do acompanhamento multiprofissional nas fases do pré, trans e pós-CB, com a finalidade de promoção à saúde, prevenção e controle de agravos (Nascimento, Bezerra, \& Angelim, 2013; Conselho Federal de Medicina [CFM], 2015; Associação Brasileira para o Estudo da Obesidade e da Síndrome Metabólica [ABESO], (2016); Barroso et al., 2017; Ribeiro, Giapietro, Belarmino, \& Salgado-Junior, 2018).

Outra questão a ser destacada é o abandono das instruções de profissionais no período pós-cirúrgico, quando o paciente pode crer que seus problemas estarão resolvidos. As mudanças acontecidas após a cirurgia são muitas, abrangendo o nível comportamental, físico e psíquico. Pode-se evitar essas surpresas destacando-se a necessidade da atuação da equipe multiprofissional em todas as fases que envolvem a cirurgia, pois o procedimento cirúrgico isolado não resolverá todos os problemas do paciente (Cordás \& Lopes-Filho; Segal, 2004).

Por fim, apenas uma mulher, neste estudo, relatou diagnóstico de HAS após a CB, reforçando o que os estudos trazem sobre a importância do procedimento cirúrgico para a redução de comorbidades associadas à obesidade, como a HAS, DM e distúrbios do sono (Mota, Costa, \& Almeida, 2014).

Este estudo apresentou algumas limitações, como o fato das mulheres terem sido avaliadas em períodos distintos após a realização da CB, além do uso de dados obtidos via telefone. Entretanto, as mulheres estão sendo acompanhadas por um longo período pós-operatório, podendo oferecer importantes informações de diversos momentos após o procedimento cirúrgico. Além disso, a coleta de dados pelo método do autorrelato tem sido amplamente utilizada como método aceitável e válido em estudos epidemiológicos com adultos brasileiros.

A potencialidade do estudo está na utilização de uma amostra de mulheres acompanhadas por um longo período em relação à importantes variáveis da gravidez e do pós-parto após o procedimento de cirurgia bariátrica em uma instituição de saúde específica, um hospital privado.

\section{Considerações Finais}

Este estudo demonstrou que o procedimento de cirurgia bariátrica pode impactar os aspectos relacionados à gravidez e pós-parto das mulheres.

Desse modo, fatores sociodemográficos, gineco-obstétricos, clínicos e comportamentais de mulheres no período da gestação e do pós-parto podem ser otimizados pelo procedimento cirúrgico, destacando a relevância dos fatores individuais e o acompanhamento multiprofissional nos períodos que englobam o pré, trans e pós cirurgia.

O procedimento de CB está relacionado a desfechos positivos, como a melhoria das condições clínicas associadas à obesidade, do desempenho sexual, da fertilidade e do prognóstico obstétrico, portanto, a manutenção destes fatores está 
relacionada à forma de encarar os novos hábitos de vida necessários após o procedimento cirúrgico e o acompanhamento multiprofissional. Apesar dos benefícios comprovados do procedimento cirúrgico, o mesmo pode levar a complicações clínicas e nutricionais maternas e fetais.

Os resultados deste estudo oferecem importantes subsídios para novas pesquisas e para a elaboração de estratégias que visem a otimização dos fatores relacionados à gravidez e pós-parto de mulheres submetidas ao procedimento de CB.

Estabelecer uma gestação mais saudável e um período pós-parto com fatores que otimizam as condições de vida e de saúde da mulher e do bebê, requer a compreensão das mudanças que o procedimento pode desencadear e na essência do acompanhamento multiprofissional.

Ressalta-se que as mulheres submetidas à CB podem se relacionar de modo diferente com a gestação em relação àquelas que nunca se submeteram à cirurgia, devido ao fato de cada mulher ter demandas, qualidades clínicas e comportamentos individuais.

Reflete-se a relevância de estudos futuros com a abrangência de variáveis relacionadas à consciência das mulheres em relação à importância do acompanhamento multiprofissional nos períodos pré, trans e pós-cirúrgico, bem como a consideração de fatores associados à melhoria dos aspectos relacionados à gravidez e pós-parto dessas mulheres.

\section{Referências}

Amaral, O., \& Pereira, C. (2016). Obesidade da genética ao ambiente. In: Millenium-Journal of Education, Technologies, and Health, 34, 311-322.

Abeso. (2016). Associação Brasileira para o Estudo da Obesidade e da Síndrome Metabólica. Diretrizes brasileiras de obesidade. (4a ed.).

Barroso, M. F. R., et al. (2017). Caracterização sócio demográfica e clínica de pacientes submetidos à cirurgia bariátrica. Rev Pesq Saúde, 18 (2), 86-90.

Beleli, C. A. V. et al. (2011). A perda de peso prevista em pacientes submetidos à derivação gástrica em Y-de-Roux. BMI Bariatricae Metabolic Ibero-American, $1(1), 16-23$.

Brasil. (2020). Ministério da Saúde. Secretaria de Atenção Primária à Saúde (SAPS). Saúde prepara ações para controle do excesso de peso e da obesidade. Brasília: Ministério da Saúde.

Brasil. (2020). Ministério da Saúde. Sistema de Vigilância Alimentar e Nutricional - (SISVAN). Notas Técnicas. Estado nutricional de adultos (Est nut adulto). Brasília: Ministério da Saúde.

Brasil. (2013). Ministério da Saúde. Portaria n 424, de 19 de março de 2013. Redefine as diretrizes para a organização da prevenção e do tratamento do sobrepeso e obesidade como linha de cuidado prioritária da Rede de Atenção à Saúde das Pessoas com Doenças Crônicas. Diário Oficial da República Federativa do Brasil, Brasília: Ministério da Saúde.

Coelho, A. et al. (2020). Pregnancy after bariatric surgery. Acta Obstet Ginecol Port., 14 (2), 106-110.

Cfm. (2015). Conselho federal de medicina. Resolução CFM no 2.131/2015. Brasília.

Cordas, T. A.; Lopes Filho, A. P., \& Segal, A. (2004). Transtorno alimentar e cirurgia bariátrica: relato de caso. Arq Bras Endocrinol Metab, 48 (4).

Costa, A et al. (2020). Recomendações para contraceção em mulheres com excesso de peso/obesidade, antes e após cirurgia bariática. Acta Obstet Ginecol Port., $14(1), 38-43$.

Eppel, D., \& Christian, S. G. (2020). Gravidez após cirurgia bariátrica: um desafio crescente na medicina feto-materna. The American Journal of Clinical Nutrition, 111 (2), 250-251.

Falcone, V. et al. (2018). Pregnancy after bariatric surgery: a narrative literature review and discussion of impact on pregnancy management and outcome. BMC pregnancy and childbirth, 18 (1), 1-13.

Febrasgo. (2014). Federação brasileira das associações de ginecologia e obstetrícia. Manual de Assistência Pré-Natal. São Paulo: FEBRASGO. 
Ferrer, F. et al. (2016). Cirugía bariátrica antes del embarazo y su impacto en el resultado perinatal; revisión de la literatura. Ars. Medica, 41 (2).

Franques, A.R. et al. (2011). O reganho de peso após a cirurgia bariátrica. Em: A. R. M. Franques, \& M. S. A. Arenales-Loli. (Org.). Novos corpos, novas realidades: reflexões sobre o pós-operatório de cirurgia da obesidade (pp. 263-272). São Paulo: Vetor.

Hruby, A., \& Hu, F. B. (2015). The epidemiology of obesity: a big picture. Pharmaco Economics, 33 (7), 673-689.

Johansson, K. et al. (2015). Outcomes of Pregnancy after Bariatric Surgery. New England Journal of Medicine, 372 (9), 814-824.

Lesko, J., \& Peaceman, A. (2012). Pregnancy Outcomes in Women After Bariatric Surgery Compared With Obese and Morbidly Obese Controls. Obstetrics \& Gynecology, 119 (3), 547-554.

Lopes, V. P. et al. (2019). Índice de massa corporal e aptidão física em adolescentes brasileiros. J. Pediatr., 95 (3), $358-365$.

Major, P. et al. (2015). Quality of Life After Bariatric Surgery. ObesSurg., 25 (9), 1703-10.

Malta, D. C. et al. (2019). Tendência temporal da prevalência de obesidade mórbida na população adulta brasileira entre os anos de 2006 e 2017 . Cadernos de Saúde Pública, 35.

Mariano, M. L. L. et al. (2014). Bariatric surgery: impact on sexuality of the obese person. Revista do Colégio Brasileiro de Cirurgiões, 41 (6), $412-420$.

Melo, F. L. E., \& Melo, M. (2017). Impacto da cirurgia bariátrica na fertilidade feminina. Reprod. Clim., 32 (1), 57-62.

Mota, D. C. L., Costa, T. M. B., \& Almeida, S. S. (2014). Imagem corporal, ansiedade e depressão em mulheres submetidas à cirurgia bariátrica. Revista Psicologia: Teoria e Prática, 16 (3), 100-113.

Nascimento, C. A. D., Bezerra, S. M. M. S., \& Angelim, E. M. S. (2013). Vivência da obesidade e do emagrecimento em mulheres submetidas à cirurgia bariátrica. Estudos de Psicologia, 18 (2), 193-201.

Oliveira, L. S. F. et al. (2018). Repercussões da cirurgia bariátrica na qualidade de vida de pacientes com obesidade: uma revisão integrativa. Revista Brasileira de Obesidade, Nutrição e Emagrecimento, 12 (69), 47-58.

Oliveira, M. A. P., \& Parente, R. C. M. (2010). Estudos de coorte e de caso-controle na Era da Medicina Baseada em Evidência. Brazilian Journal of Videoendoscopic Surgery, 3 (3), 115-125.

Pereira, A. S. et al. (2018). Metodologia da pesquisa científica. UFSM. https://repositorio.ufsm.br/bitstream/handle/1/15824/Lic_Computacao_MetodologiaPesquisa-Cientifica.pdf?sequence $=1$.

Petrucciani, N. et al. (2020). Management of surgical complications of previous bariatric surgery in pregnant women. A systematic review from the BARIAMAT Study Group. Surgery for Obesity and Related Diseases, 16 (2), 312-331.

Pinheiro, R. C. M. et al. (2014). Implicações nutricionais da gravidez após cirurgia debypass gástrico: uma revisão da literatura. Comun. ciênc. saúde, 69-78.

Poitou Bernert, C. et al. (2007). Nutritional deficiency after gastric bypass: diagnosis, prevention, treatment. Diabetes \& Metabolism, 33 , $13-24$.

Raoof, M. et al. (2015). Health-Related Quality-of-Life (HRQoL) on an Average of 12 Years After Gastric Bypass Surgery. Obes Surg., 25 (7), $1119-27$.

Ribeiro, G. A. N. A. et al. (2018). Depression, anxiety, and binge eating before and after bariatric surgery: Problems thatremain. ABCD Arq Bras Cir Dig., 31 (1).

Rosales, J. J. B. et al. (2020). Gravidez pós cirurgia bariátrica: uma revisão integrativa. Revista Eletrônica Acervo Saúde, 12 (2).

Roos, N. et al. (2013). Perinatal outcomes after bariatric surgery: nationwide Br population based matched cohort study. BMJ, 347 (12), $6460-6460$.

Różańska-Walędziak, A. et al. (2020). The Influence of Bariatric Surgery on Pregnancy and Perinatal Outcome-A Case-Control Study. Journal of Clinical Medicine, 9 (5), 1324.

Sbcbm. (2018). Sociedade Brasileira de Cirurgia Bariátrica e Metabólica. Número de cirurgias bariátricas no Brasil aumenta 46,7\%. Recuperado de: https://www.sbcbm.org.br/numero-de-cirurgias-bariatricas-no-brasil-aumenta-467/.

Sheiner, E. et al. (2004). Pregnancy after bariatric surgery is not associated with adverse perinatal outcome. Am J Obstet Gynecol., 190 (5), $1335-40$.

Sousa, K. O., \& Johann, R.L.V.O. (2014). Cirurgia bariátrica e qualidade de vida. Psicol. Argum., 32 (79), $155-64$.

Stata. (2020). Analysis and Statistical Software. https://www.stata.com/stata14/.

Teste Shapiro Wilk. (2020). http://www.portalaction.com.br/inferencia/64-teste-de-shapiro-wilk.

Wojcicki, J. M. (2011). Maternal prepregnancy body mass index and initiation and duration of breastfeeding: a review of the literature. $J$ Womens Health, 20 (3), 341-7. 